\title{
Rapid Particle Swarm Optimization Algorithm for Solving Optimal Reactive Power Dispatch Problem
}

\author{
Kanagasabai Lenin ${ }^{1^{*}}$, B.Ravindhranath Reddy ${ }^{2}$, M. Surya Kalavathi ${ }^{3}$ \\ Electrical and Electronics Engineering, Jawaharlal Nehru Technological University Kukatpally, \\ Hyderabad 500 085, India. \\ e-mail: gklenin@gmail.com
}

\begin{abstract}
In this paper Rapid Particle Swarm Optimization (RPSO) algorithm is proposed to solve the optimal reactive power dispatch Problem. The Rapid Particle swarm Optimization (RPSO) algorithm is obtained by merging PSO with Cauchy mutation. Basic idea is to introduce the Cauchy mutation into PSO such that it prevents PSO from trapping into a local optimum through stretched jumps made by the Cauchy mutation. In order to evaluate the efficiency of the proposed algorithm, it has been tested on IEEE 30 bus system and compared other standard algorithms. Results show's that RPSO is more efficient in reducing the real power loss and voltage index also improved
\end{abstract}

Keywords: rapid particle swarm optimization, cauchy mutation, optimal reactive power, transmission loss

\section{Introduction}

In recent years the optimal reactive power dispatch (ORPD) problem has received great attention as a result of the improvement on economy and security of power system operation. Solutions of ORPD problem aim to minimize object functions such as fuel cost, power system loses, etc. while satisfying a number of constraints like limits of bus voltages, tap settings of transformers, reactive and active power of power resources and transmission lines and a number of controllable Variables [1,2]. In the literature, many methods for solving the ORPD problem have been done up to now. At the beginning, several classical methods such as gradient based [3], interior point [4], linear programming [5] and quadratic programming [6] have been successfully used in order to solve the ORPD problem. However, these methods have some disadvantages in the Process of solving the complex ORPD problem. Drawbacks of these algorithms can be declared insecure convergence properties, long execution time, and algorithmic complexity. Besides, the solution can be trapped in local minima [1,7]. In order to overcome these disadvantages, researches have successfully applied evolutionary and heuristic algorithms such as Genetic Algorithm (GA) [2], Differential Evolution (DE) [8] and Particle Swarm Optimization (PSO) [9]. It is reported in those that evolutionary or heuristic algorithms are more efficient than classical algorithms for solving the reactive power problem. During the last decades a lot of population-based Meta heuristic algorithms were proposed. Voltage stability evaluation using modal analysis [10] is used as the indicator of voltage stability. In the recent decades a number of optimization algorithms based on natural phenomena have been developed. Particle Swarm Optimization (PSO) [11-18] is motivated from the social behaviour of organisms, such as bird flocking and fish schooling. In order to prevent PSO from falling in a local optimum, a Rapid PSO (RPSO) is proposed by integrating a Cauchy mutation operator. Because the expectation of Cauchy distribution does not exist, the variance of Cauchy distribution is infinite. Some researches [19-20] have indicated that the Cauchy mutation operator is good at the global search for its long jump ability. Besides the Cauchy mutation, RPSO chooses the natural selection strategy of evolutionary algorithms as the basic elimination strategy of particles. RPSO combines PSO with Cauchy mutation and evolutionary selection strategy. It has the fast convergence speed characteristic of PSO, and greatly overcomes the tendency of trapping into local optima of PSO.The performance of RPSO has been evaluated in standard IEEE 30 bus test system and the results analysis shows that our proposed approach outperforms all approaches investigated in this paper. 


\section{Voltage Stability Evaluation}

\subsection{Modal analysis for voltage stability evaluation}

Modal analysis is one among best methods for voltage stability enhancement in power systems. The steady state system power flow equations are given by.

$$
\left[\begin{array}{l}
\Delta \mathrm{P} \\
\Delta \mathrm{Q}
\end{array}\right]=\left[\begin{array}{cc}
\mathrm{J}_{\mathrm{p} \theta} & \mathrm{J}_{\mathrm{pv}} \\
\mathrm{J}_{\mathrm{q} \theta} & \mathrm{J}_{\mathrm{QV}}
\end{array}\right]\left[\begin{array}{l}
\Delta \theta \\
\Delta \mathrm{V}
\end{array}\right]
$$

where :

$\Delta \mathrm{P}=$ Incremental change in bus real power.

$\Delta Q=$ Incremental change in bus reactive Power injection

$\Delta \theta=$ incremental change in bus voltage angle.

$\Delta \mathrm{V}=$ Incremental change in bus voltage Magnitude

$\mathrm{Jp} \theta, \mathrm{JPV}, \mathrm{JQ} \theta, \mathrm{JQV}$ jacobian matrix are the sub-matrixes of the System voltage stability is affected by both $P$ and $Q$. To reduce (1), let $\Delta P=0$, then.

$$
\begin{aligned}
& \Delta \mathrm{Q}=\left[\mathrm{J}_{\mathrm{QV}}-\mathrm{J}_{\mathrm{Q} \theta} \mathrm{J}_{\mathrm{P} \theta^{-1}} \mathrm{~J}_{\mathrm{PV}}\right] \Delta \mathrm{V}=\mathrm{J}_{\mathrm{R}} \Delta \mathrm{V} \\
& \Delta \mathrm{V}=\mathrm{J}^{-1}-\Delta \mathrm{Q} \\
& \text { Where } \\
& \mathrm{J}_{\mathrm{R}}=\left(\mathrm{J}_{\mathrm{QV}}-\mathrm{J}_{\mathrm{Q} \theta} \mathrm{J}_{\mathrm{P}^{-1}} \mathrm{JPV}\right)
\end{aligned}
$$

$\mathrm{J}_{\mathrm{R}}$ is called the reduced Jacobian matrix of the system.

\subsection{Modes of Voltage instability:}

Voltage Stability characteristics of the system have been identified by computing the Eigen values and Eigen vectors.

$$
\begin{aligned}
& \text { Let } \\
& \mathrm{J}_{\mathrm{R}}=\xi \wedge \eta
\end{aligned}
$$

where,

$$
\begin{aligned}
& \xi=\text { right eigenvector matrix of } J R \\
& \eta=\text { left eigenvector matrix of } J R \\
& \Lambda=\text { diagonal eigenvalue matrix of } J R \text { and } \\
& J_{R^{-1}}=\xi \Lambda^{-1} \eta
\end{aligned}
$$

From (5) and (8), we have

$$
\begin{gathered}
\Delta \mathrm{V}=\xi \wedge^{-1} \eta \Delta \mathrm{Q} \\
\text { Or } \\
\Delta \mathrm{V}=\sum_{\mathrm{I}} \frac{\xi_{\mathrm{i}} \eta_{\mathrm{i}}}{\lambda_{\mathrm{i}}} \Delta \mathrm{Q}
\end{gathered}
$$

where $\xi i$ is the ith column right eigenvector and $\eta$ the ith row left eigenvector of $J R$. $\lambda i$ is the ith Eigen value of JR. The ith modal reactive power variation is,

$$
\Delta \mathrm{Q}_{\mathrm{mi}}=\mathrm{K}_{\mathrm{i}} \xi_{\mathrm{i}}
$$

where,

$$
\mathrm{K}_{\mathrm{i}}=\sum_{\mathrm{j}} \xi_{\mathrm{ij}}-1
$$


where

$\xi j i$ is the jth element of $\xi i$

The corresponding ith modal voltage variation is

$\Delta \mathrm{V}_{\mathrm{mi}}=\left[1 / \lambda_{\mathrm{i}}\right] \Delta \mathrm{Q}_{\mathrm{mi}}$

If $|\lambda \mathrm{i}|=0$ then the ith modal voltage will collapse.

Then,

In (10), let $\Delta Q=$ ek where ek has all its elements zero except the kth one being 1 .

$\Delta \mathrm{V}=\sum_{\mathrm{i}} \frac{\eta_{1 \mathrm{k}} \xi_{1}}{\lambda_{1}}$

$\eta_{1 \mathrm{k}} \quad k$ th element of $\eta_{1}$

$\mathrm{V}-\mathrm{Q}$ sensitivity at bus $\mathrm{k}$

$\frac{\partial \mathrm{V}_{\mathrm{K}}}{\partial \mathrm{Q}_{\mathrm{K}}}=\sum_{\mathrm{i}} \frac{\mathrm{n}_{1 \mathrm{k}} \xi_{1}}{\lambda_{1}}=\sum_{\mathrm{i}} \frac{\mathrm{P}_{\mathrm{ki}}}{\lambda_{1}}$

\section{Problem Formulation}

The objectives of the reactive power dispatch problem is to minimize the system real power loss and maximize the static voltage stability margins (SVSM).

\subsection{Minimization of Real Power Loss}

Minimization of the real power loss (Ploss) in transmission lines is mathematically stated as follows.

$$
\mathrm{P}_{\text {loss }}=\sum_{\mathrm{k}=(\mathrm{i}, \mathrm{j})}^{\mathrm{n}=1} \mathrm{~g}_{\mathrm{k}\left(\mathrm{V}_{\mathrm{i}}^{2}+\mathrm{V}_{\mathrm{j}}^{2}-2 \mathrm{~V}_{\mathrm{i}} \mathrm{V}_{\mathrm{j}} \cos \theta_{\mathrm{ij}}\right)}
$$

where $\mathrm{n}$ is the number of transmission lines, $\mathrm{gk}$ is the conductance of branch $\mathrm{k}, \mathrm{Vi}$ and $\mathrm{Vj}$ are voltage magnitude at bus $i$ and bus $j$, and $\theta i j$ is the voltage angle difference between bus $i$ and bus j.

\subsection{Minimization of Voltage Deviation}

Minimization of the voltage deviation magnitudes (VD) at load buses is mathematically stated as follows.

Minimize VD $=\sum_{\mathrm{k}=1}^{\mathrm{nl}}\left|\mathrm{V}_{\mathrm{k}}-1.0\right|$

Where $\mathrm{nl}$ is the number of load busses and Vk is the voltage magnitude at bus $\mathrm{k}$.

\subsection{System Constraints}

Objective functions are subjected to these constraints shown below. Load flow equality constraints:

$$
\begin{aligned}
& P_{G i}-P_{D i}-V_{i} \sum_{j=1}^{n b} v_{j}\left[\begin{array}{cc}
G_{i j} & \cos \theta_{i j} \\
+B_{i j} & \sin \theta_{i j}
\end{array}\right]=0, i=1,2 \ldots, n b \\
& Q_{G i}-Q_{D i}-V_{i} \sum_{j=1}^{n b} v_{j}\left[\begin{array}{cc}
G_{i j} & \cos \theta_{i j} \\
+B_{i j} & \sin \theta_{i j}
\end{array}\right]=0, i=1,2 \ldots, n b
\end{aligned}
$$

where, $\mathrm{nb}$ is the number of buses, $\mathrm{PG}$ and $\mathrm{QG}$ are the real and reactive power of the generator, $\mathrm{PD}$ and $\mathrm{QD}$ are the real and reactive load of the generator, and Gij and Bij are the mutual conductance and susceptance between bus $i$ and bus $j$.

Generator bus voltage (VGi) inequality constraint:

$\mathrm{V}_{\mathrm{Gi}}^{\min } \leq \mathrm{V}_{\mathrm{Gi}} \leq \mathrm{V}_{\mathrm{Gi}}^{\max }, \mathrm{i} \in \mathrm{ng}$

Load bus voltage (VLi) inequality constraint: 


$$
V_{\mathrm{Li}}^{\min } \leq \mathrm{V}_{\mathrm{Li}} \leq \mathrm{V}_{\mathrm{Li}}^{\max }, \mathrm{i} \in \mathrm{nl}
$$

Switchable reactive power compensations (QCi) inequality constraint:

$$
\mathrm{Q}_{\mathrm{Ci}}^{\min } \leq \mathrm{Q}_{\mathrm{Ci}} \leq \mathrm{Q}_{\mathrm{Ci}}^{\max }, \mathrm{i} \in \mathrm{nc}
$$

Reactive power generation (QGi) inequality constraint:

$\mathrm{Q}_{\mathrm{Gi}}^{\min } \leq \mathrm{Q}_{\mathrm{Gi}} \leq \mathrm{Q}_{\mathrm{Gi}}^{\max }, \mathrm{i} \in \mathrm{ng}$

Transformers tap setting (Ti) inequality constraint:

$$
\mathrm{T}_{\mathrm{i}}^{\min } \leq \mathrm{T}_{\mathrm{i}} \leq \mathrm{T}_{\mathrm{i}}^{\max }, \mathrm{i} \in \mathrm{nt}
$$

Transmission line flow (SLi) inequality constraint:

$$
S_{L i}^{\min } \leq S_{L i}^{\max }, i \in n l
$$

where, nc, ng and $\mathrm{nt}$ are numbers of the switchable reactive power sources, generators and transformers

\section{Particle Swarm Optimization (PSO)}

Particle Swarm Optimization Algorithm (PSO) is a population based optimization tool, where the system is initialized with a population of random particles and the algorithm searches for optima by updating generations. Suppose that the search space is D-dimensional. The position of the $i$-th particle can be represented by a D-dimensional vector $X_{i}=\left(x_{i 1}, x_{i 2}, \ldots, x_{i D}\right)$ and the velocity of this particle is $\mathrm{V}_{\mathrm{i}}=\left(\mathrm{v}_{\mathrm{i} 1}, \mathrm{v}_{\mathrm{i} 2}, \ldots, \mathrm{v}_{\mathrm{iD}}\right)$. The best previously visited position of the $\mathrm{i}$ th particle is represented by $P_{i}=\left(p_{i 1}, p_{i 2}, \ldots, p_{i D}\right)$ and the global best position of the swarm found so far is denoted by $P_{g}=\left(p_{g 1}, p_{g 2}, \ldots, p_{g D}\right)$. The fitness of each particle can be evaluated through putting its position into a designated objective function. The particle's velocity and its new position are updated as follows:

$$
\begin{aligned}
& v_{i d}^{t+1}=\omega^{t} v_{i d}^{t}+c_{1} r_{1}^{t}\left(p_{i d}^{t}-x_{i d}^{t}\right)+c_{2} r_{2}^{t}\left(p_{g d}^{t}-x_{i d}^{t}\right) \\
& x_{i d}^{t+1}=x_{i d}^{t}+v_{i d}^{t+1}
\end{aligned}
$$

where $d \in\{1,2, \ldots, D\}, i \in\{1,2, \ldots, N\} N$ is the population size, the superscript $t$ denotes the iteration number, $\omega$ is the inertia weight, $r 1$ and $r 2$ are two random values in the range $[0,1], c 1$ and $\mathrm{c} 2$ are the cognitive and social scaling parameters which are positive constants.

\section{Cauchy Mutation Merged into PSO}

From the mathematic theoretical analysis of the trajectory of a PSO particle [21-23], the trajectory of a particle Xid converges to a weighted mean of Pid and Pgd. Whenever the particle converges, it will "fly" to the personal best position and the global best particle's position. According to the update equation, the personal best position of the particle will gradually move closer to the global best position. Therefore, all the particles will converge onto the global best particle's position. This information sharing mechanism makes PSO have a very fast speed of convergence. Meanwhile, because of this mechanism, PSO can't guarantee to find the global minimal value of a function. In fact, the particles usually converge to local optima. Without loss of generality, only function minimization is discussed here. Once the particles trap into a local optimum, in which Pid can be assumed to be the same as Pgd, all the particles converge on Pgd. At this condition, the velocity update equation becomes:

$$
V_{\mathrm{id}}^{\prime}=\omega V_{\mathrm{id}}
$$

IJEEI Vol. 5, No. 2, June 2017 : $99-107$ 
When the iteration in the equation (26) goes to infinite, the velocity of the particle Vid will be close to 0 because of $0 \leq \omega<1$. After that, the position of the particle Xid will not change, so that PSO has no capability of jumping out of the local optimum. It is the reason that PSO often fails on finding the global minimal value. To overcome the weakness of PSO discussed at the beginning of this section, the Cauchy mutation is incorporated into PSO algorithm. The basic idea is that, the velocity and positions of a particle are updated not only according to (24) and (25), but also according to Cauchy mutation as follows:

$$
\begin{aligned}
& \mathrm{V}_{\mathrm{id}}^{\prime}=\mathrm{V}_{\mathrm{id}} \exp (\delta) \\
& \mathrm{X}_{\mathrm{id}}^{\prime}=\mathrm{X}_{\mathrm{id}}+\mathrm{V}_{\mathrm{id}}^{\prime} \delta_{\mathrm{id}}
\end{aligned}
$$

where $\delta$ and $\delta$ id denote Cauchy random numbers since the expectation of Cauchy distribution doesn't exist, the variance of Cauchy distribution is infinite so that Cauchy mutation could make a particle have a long jump. By adding the update equations of (27) and (28), RPSO greatly increases the probability of escaping from the local optimum. In standard PSO, the position of a particle is updated according to equations (24) and (25). That is, for each particle there is nowhere to move but following the direction of the best particle and the flying direction is nearly determinate through the generation. From the above analysis of PSO, the particles incline to converge on a local optimum.

\subsection{Natural Selection Strategy}

In the standard PSO, all particles are directly updated by their offspring no matter whether they are improved. If a particle moves to a better position, it can be replaced by the updated. However if it moves to a worse position, it is still replaced by its offspring. In fact, the most particles fly to worse positions for most cases; therefore the whole swarm will converge on local optima. Like evolutionary algorithms, RPSO introduces an evolutionary selection strategy in which each particle survives according to a natural selection rule. Therefore, the particle's position at the next step is not only due to the position update but also the evolutionary selection. Such strategy could greatly reduce the probability of trapping into local optimum. The evolutionary selection strategy is carried out as follows. Assume the size of the swarm is $\mathrm{m}$, pair-wise comparison over the union of parents and offspring $(1,2, \ldots 2 \mathrm{~m})$ is made. For each particle, q opponents are randomly chosen from all parents and offspring with equal probability. If the fitness of particle $i$ is less than its opponent, it will receive a "win". Then select $m$ particles that have the more winnings to be the next generation.

The detail of the selection framework is as follows:

a. Step1: For each particle of parent and offspring, assign win[i $]=0$.

b. Step2: Randomly select q particles (opponents) for each particle in parent and offspring.

c. Step3: For each particle, compare it with its q opponents. For particle i, if the fitness of its opponent $\mathrm{j}$ is larger than particle $\mathrm{i}$, then win[i]++.

d. Step4: Select $\mathrm{m}$ particles that have the more winnings to be the next generation.

RPSO Algorithm for solving reactive power dispatch problem

1. Produce the preliminary particles by arbitrarily producing the position and velocity for each particle.

2. Appraise each particle's fitness.

3. For each particle, if its fitness is smaller than its previous best $\left(P_{i d}\right)$ fitness, update $P_{\text {id }}$.

4. For each particle, if its fitness is smaller than the best one $\left(P_{g d}\right)$ of all the particles, update Pgd.

5. For each particle, do

a) Engender a new particle $t$ according to the formula (24) and (25).

b) Engender a new particle t' according to the formula (27) and (28).

c) Compare $t$ with $t$ 'chose the one with smaller fitness to be the offspring.

6. Produce the next generation according to the above evolutionary selection strategy.

7. If end criterion is satisfied, then stop, otherwise go to 3 . 


\section{Simulation Results}

The efficiency of the proposed Rapid Particle Swarm Optimization (RPSO) is demonstrated by testing it on standard IEEE-30 bus system. The IEEE-30 bus system has 6 generator buses, 24 load buses and 41 transmission lines of which four branches are (6-9), (6$10),(4-12)$ and (28-27) - are with the tap setting transformers. The lower voltage magnitude limits at all buses are 0.95 p.u. and the upper limits are 1.1 for all the PV buses and 1.05 p.u. for all the $P Q$ buses and the reference bus. The simulation results have been presented in Tables 1, 2, 3 \&4. And in the Table 5 shows the proposed algorithm powerfully reduces the real power losses when compared to other given algorithms. The optimal values of the control variables along with the minimum loss obtained are given in Table 1. Corresponding to this control variable setting, it was found that there are no limit violations in any of the state variables.

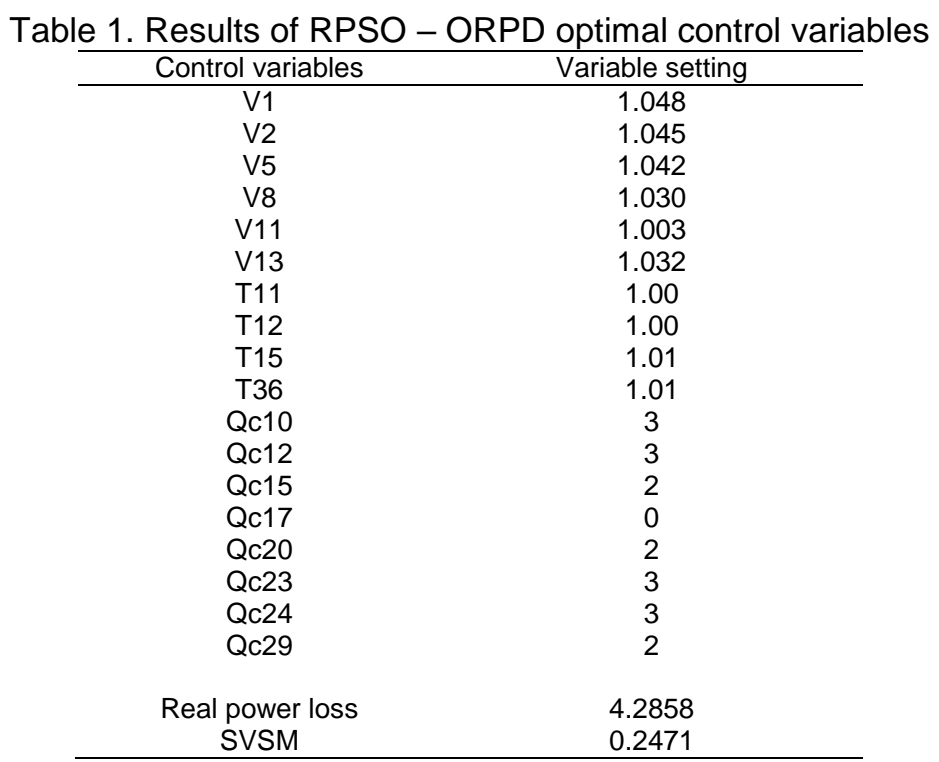

Optimal Reactive Power Dispatch problem together with voltage stability constraint problem was handled in this case as a multi-objective optimization problem where both power loss and maximum voltage stability margin of the system were optimized simultaneously. Table 2 indicates the optimal values of these control variables. Also it is found that there are no limit violations of the state variables. It indicates the voltage stability index has increased from 0.2471 to 0.2486 , an advance in the system voltage stability. To determine the voltage security of the system, contingency analysis was conducted using the control variable setting obtained in case 1 and case 2. The Eigen values equivalents to the four critical contingencies are given in Table 3. From this result it is observed that the Eigen value has been improved considerably for all contingencies in the second case. 
Table 2. Results of RPSO -Voltage Stability Control Reactive Power Dispatch Optimal Control Variables

\begin{tabular}{cc}
\hline Control Variables & Variable Setting \\
\hline V1 & 1.048 \\
V2 & 1.046 \\
V5 & 1.045 \\
V8 & 1.031 \\
V11 & 1.004 \\
V13 & 1.033 \\
T11 & 0.090 \\
T12 & 0.090 \\
T15 & 0.090 \\
T36 & 0.090 \\
Qc10 & 3 \\
Qc12 & 3 \\
Qc15 & 2 \\
Qc17 & 3 \\
Qc20 & 0 \\
Qc23 & 2 \\
Qc24 & 2 \\
Qc29 & 3 \\
Real power loss & 4.9899 \\
SVSM & 0.2486 \\
\hline
\end{tabular}

Table 3. Voltage Stability under Contingency State

\begin{tabular}{cccc}
\hline SI.No & Contingency & ORPD Setting & VSCRPD Setting \\
\hline 1 & $28-27$ & 0.1419 & 0.1434 \\
2 & $4-12$ & 0.1642 & 0.1650 \\
3 & $1-3$ & 0.1761 & 0.1772 \\
4 & $2-4$ & 0.2022 & 0.2043 \\
\hline
\end{tabular}

Table 4. Limit Violation Checking Of State Variables

\begin{tabular}{ccccc}
\hline State & \multicolumn{2}{c}{ limits } & ORPD & VSCRPD \\
variables & Lower & upper & & \\
\hline Q1 & -20 & 152 & 1.3422 & -1.3269 \\
Q2 & -20 & 61 & 8.9900 & 9.8232 \\
Q5 & -15 & 49.92 & 25.920 & 26.001 \\
Q8 & -10 & 63.52 & 38.8200 & 40.802 \\
Q11 & -15 & 42 & 2.9300 & 5.002 \\
Q13 & -15 & 48 & 8.1025 & 6.033 \\
V3 & 0.95 & 1.05 & 1.0372 & 1.0392 \\
V4 & 0.95 & 1.05 & 1.0307 & 1.0328 \\
V6 & 0.95 & 1.05 & 1.0282 & 1.0298 \\
V7 & 0.95 & 1.05 & 1.0101 & 1.0152 \\
V9 & 0.95 & 1.05 & 1.0462 & 1.0412 \\
V10 & 0.95 & 1.05 & 1.0482 & 1.0498 \\
V12 & 0.95 & 1.05 & 1.0400 & 1.0466 \\
V14 & 0.95 & 1.05 & 1.0474 & 1.0443 \\
V15 & 0.95 & 1.05 & 1.0457 & 1.0413 \\
V16 & 0.95 & 1.05 & 1.0426 & 1.0405 \\
V17 & 0.95 & 1.05 & 1.0382 & 1.0396 \\
V18 & 0.95 & 1.05 & 1.0392 & 1.0400 \\
V19 & 0.95 & 1.05 & 1.0381 & 1.0394 \\
V20 & 0.95 & 1.05 & 1.0112 & 1.0194 \\
V21 & 0.95 & 1.05 & 1.0435 & 1.0243 \\
V22 & 0.95 & 1.05 & 1.0448 & 1.0396 \\
V23 & 0.95 & 1.05 & 1.0472 & 1.0372 \\
V24 & 0.95 & 1.05 & 1.0484 & 1.0372 \\
V25 & 0.95 & 1.05 & 1.0142 & 1.0192 \\
V26 & 0.95 & 1.05 & 1.0494 & 1.0422 \\
V27 & 0.95 & 1.05 & 1.0472 & 1.0452 \\
V28 & 0.95 & 1.05 & 1.0243 & 1.0283 \\
V29 & 0.95 & 1.05 & 1.0439 & 1.0419 \\
V30 & 0.95 & 1.05 & 1.0418 & 1.0397 \\
\hline & & & &
\end{tabular}


Table 5. Comparison of Real Power Loss

\begin{tabular}{ll}
\hline \multicolumn{1}{c}{ Method } & Minimum loss \\
\hline Evolutionary programming [24] & 5.0159 \\
Genetic algorithm [25] & 4.665 \\
Real coded GA with Lindex as SVSM [26] & 4.568 \\
Real coded genetic algorithm [27] & 4.5015 \\
Proposed RPSO method & 4.2858 \\
\hline
\end{tabular}

\section{Conclusion}

In this paper, one of the recently developed Rapid Particle Swarm Optimization (RPSO) has been applied to solve optimal reactive power dispatch problem. Different objective functions have been utilized to minimize real power loss and the voltage profile has been improved. Projected RPSO approach has been tested on the IEEE 30-bus power system \& simulation results indicate the effectiveness and robustness of the proposed RPSO algorithm in solving optimal reactive power dispatch problem.

\section{References}

[1] MA Abido, JM Bakhashwain. A novel multi objective evolutionary algorithm for optimal reactive power dispatch problem. in proc. Electronics, Circuits and Systems conf. 2003; 3: 1054-1057.

[2] WNW Abdullah, H Saibon, AAM Zain, KL Lo. Genetic Algorithm for Optimal Reactive Power Dispatch. in proc. Energy Management and Power Delivery conf. 1998; 1: 160-164.

[3] KY Lee, YM Park, JL Ortiz. Fuel-cost minimisation for both real and reactive-power dispatches. in proc. Generation, Transmission and Distribution conf. 1984; 131: 85-93.

[4] S Granville. Optimal Reactive Dispatch Trough Interior Point Methods. IEEE Trans. on Power Systems. 1994; 9: 136-146.

[5] NI Deeb, SM Shahidehpour. An Efficient Technique for Reactive Power Dispatch Using a Revised Linear Programming Approach. Electric Power System Research. 1988; 15: 121-134.

[6] N Grudinin. Reactive Power Optimization Using Successive Quadratic Programming Method. IEEE Trans. on Power Systems. 1998; 13: 1219-1225.

[7] MA Abido. Optimal Power Flow Using Particle Swarm Optimization. Electrical Power and Energy Systems. 2002; 24: 563-571.

[8] AA Abou El Ela, MA Abido, SR Spea. Differential Evolution Algorithm for Optimal Reactive Power Dispatch. Electric Power Systems Research. 2011; 81: 458-464.

[9] V Miranda, N Fonseca. EPSO-Evolutionary Particle Swarm Optimization, A New Algorithm with Applications in Power Systems. in Proc. of Transmission and Distribution conf. 2002; 2: 745-750.

[10] CA Canizares, ACZ de Souza and VH Quintana. Comparison of performance indices for detection of proximity to voltage collapse. 1996;11(3): 1441-1450.

[11] J Kennedy, RC Eberhart. Particle Swarm Optimization. IEEE International Conference on Neural Networks. 1995: 1942-1948.

[12] RC Eberhart, J Kennedy. A New Optimizer Using Particle Swarm Theory. Proceedings of the 6th International Symposium on Micro Machine and Human Science. 1995: 39-43.

[13] A Ratnaweera, SK Halgamuge, HC Watson. Self-organizing hierarchical particle swarm optimizer with time-varying acceleration coefficients. IEEE Transactions on Evolutionary Computation. 2004; 8(3): 240-255.

[14] J Sun, B Feng, W Xu. Particle swarm optimization with particles having quantum behavior. in Proceedings of the IEEE Congress on Evolutionary Computation, Portland, Oregon USA. 2004: 325331.

[15] Jing Liu, Wenbo Xu, Jun Sun. Quantum-behaved particle swarm optimization with mutation operator. Proceedings of the 17th IEEE International Conference on Tools with Artificial Intelligence Pages. 2005: $237-240$.

[16] RA Krohling. Gaussian particle swarm with jumps. in Proceedings of the IEEE Congress on Evolutionary Computation. Edinburgh, UK. 2005: 1226-1231.

[17] RA Krohling, L dos Santos Coelho. PSO-E: Particle Swarm with Exponential Distribution. in Proceedings of the IEEE Congress on Evolutionary Computation. 2006: 1428- 1433.

[18] H Narihisa, T Taniguchi, M Ohta, and K Katayama. Evolutionary Programming with Exponential Mutation. in Proceedings of the IASTED Artificial Intelligence and soft Computing. Benidorn, Spain. 2005: 55-50.

[19] $\mathrm{X}$ Yao and $\mathrm{Y}$ Liu. Fast evolutionary programming. Proc. of the Fifth Annual Conference on Evolutionary Programming (EP'96). San Diego, CA, USA. 29/2-2/3/96.1996: 451-460. the MIT Press.

IJEEI Vol. 5, No. 2, June 2017: $99-107$ 
[20] X Yao, Y Liu, G Lin. Evolutionary programming made faster. IEEE Trans. Evolutionary Computation. 1999: 82-102.

[21] M Clerc and J Kennedy. The Particle Swarm: Explosion, Stability and Convergence in a MultiDimensional Complex Space. IEEE Trans. on Evolutionary Computation. 2002; 6: 58-73.

[22] G Pampara, N Franken, AP Engelbrecht. Combining Particle Swarm Optimisation with angle modulation to solve binary problems. in proceedings of the IEEE Congress on Evolutionary Computation. 2005: 89- 96.

[23] F van den Bergh. An Analysis of Particle Swarm Optimizers. PhD thesis, Department of Computer Science, University of Pretoria, South Africa. 2002.

[24] Wu QH, Ma JT. Power system optimal reactive power dispatch using evolutionary programming. IEEE Transactions on power systems. 1995;10(3): 1243-1248.

[25] S Durairaj, D Devaraj, PS Kannan. Genetic algorithm applications to optimal reactive power dispatch with voltage stability enhancement. IE(I) Journal-EL. 2006.

[26] D.Devaraj. Improved genetic algorithm for multi - objective reactive power dispatch problem. European Transactions on electrical power. 2007; 17: 569-581.

[27] P Aruna Jeyanthy, D Devaraj. Optimal Reactive Power Dispatch for Voltage Stability Enhancement Using Real Coded Genetic Algorithm. International Journal of Computer and Electrical Engineering. 2010; 2(4): 1793-8163. 